\title{
Daylight Operation of a Sodium Laser Guide Star
}

\author{
Michael Hart ${ }^{* a, c}$, Stuart Jefferies ${ }^{\mathrm{b}, \mathrm{c}}$, and Neil Murphy ${ }^{\mathrm{d}}$ \\ ${ }^{a}$ College of Optical Sciences, University of Arizona, Tucson, AZ 85721 \\ ${ }^{b}$ Department of Physics and Astronomy, Georgia State University, Atlanta, GA 30303 \\ ${ }^{c}$ Institute for Astronomy, University of Hawaii, 34 Ohia Ku Street, Pukalani, HI 96768 \\ ${ }^{\mathrm{d} J e t}$ Propulsion Laboratory, California Institute of Technology, Pasadena, CA 91109
}

\begin{abstract}
We report photometric measurements of a sodium resonance guide star against the daylight sky when observed through a tuned magneto-optical filter (MOF). The MOF comprises a sodium vapor cell in a kilogauss-level magnetic field between crossed polarizers and has a very narrow transmission profile at the sodium $\mathrm{D}_{2}$ resonance of approximately $0.008 \mathrm{~nm}$. Our observations were made with the $1.5 \mathrm{~m}$ Kuiper telescope on Mt. Bigelow, AZ, which has a separately mounted guide star laser projecting a circularly polarized single-frequency beam of approximately $6.5 \mathrm{~W}$ at $589.16 \mathrm{~nm}$. Both the beam projector and the $1.5 \mathrm{~m}$ telescope were pointed close to zenith; the baseline between them is approximately $5 \mathrm{~m}$. Measurements of the guide star were made on the morning of 2016 March 24 using an imaging camera focused on the beacon and looking through the full aperture of the telescope. The guide star flux was estimated at $1.20 \times 10^{6} \mathrm{photon} / \mathrm{m}^{2} / \mathrm{s}$ while at approximately 45 minutes after sunrise, the sky background through the MOF was 1100 photon $/ \mathrm{m}^{2} / \mathrm{s} / \operatorname{arcsec}^{2}$. We interpret our results in terms of thermal infrared observations with adaptive optics on the next generation of extremely large telescopes now being built.
\end{abstract}

Keywords: sodium laser guide star, wave-front sensing, daylight observing, extremely large telescopes, thermal infrared

\section{INTRODUCTION}

Laser guide stars (LGS) are an effective means to extend the sky coverage and scientific value of adaptive optics (AO) systems operating at astronomical telescopes. Indeed, most large observatories around the world now employ beacons generated by resonant backscatter of laser light at the $\mathrm{D}_{2}$ line of $589.16 \mathrm{~nm}$ from atomic sodium in the mesosphere. In 2001, Beckers and Cacciani ${ }^{1}$ suggested that the value of extremely large telescopes (ELTs) in the $25 \mathrm{~m}$ class and larger could be extended by using them to make high-resolution observations in thermal infrared wavelengths during the day since the limitation imposed by the bright sky background in these bands is no worse than at night. They noted that such telescopes would benefit from laser-guided AO correction at wavelengths of $3.5 \mu \mathrm{m}$ and longer, though telescopes of the time were already diffraction limited in that regime. While there are of course serious practical challenges to operating a large telescope when the sun is above the horizon, there is also a significant advantage. The scientific return on the substantial investment in ELTs, which is now being made, could be roughly doubled by operating them essentially around the clock. The three major ELT projects - the European Extremely Large Telescope ${ }^{2}$ (E-ELT), the Giant Magellan Telescope ${ }^{3}$ (GMT), and the Thirty Meter Telescope ${ }^{4}$ (TMT) - are all planning AO systems driven by signals from multiple sodium LGS and could in principle benefit from the approach. What is needed is a tool to allow the LGS to be used effectively when seen against the bright sky background at the beacon wavelength. Beckers and Cacciani noted that this could be done by placing the wave-front sensor (WFS) behind a sodium atomic vapor filter. Such filters are referred to in the literature as Faraday anomalous dispersion optical filters (FADOF) or magneto-optical filters (MOF). Here we adopt the MOF naming convention. The MOF has a number of desirable characteristics including high throughput and wavelength stability, and wide angular acceptance. Most importantly, it offers a bandpass of less than 10 $\mathrm{pm}$, which is sufficiently narrow to block almost all the scattered sunlight but wide enough to admit all the LGS light. ${ }^{5}$

Sodium laser systems designed to probe atmospheric properties through LIDAR have employed MOFs to permit daylight operation. ${ }^{6,7}$ These systems have different requirements from LGS for AO, typically using lasers with low pulse rates at tens of hertz, ${ }^{8,9}$ and great care is needed to stabilize the center frequency of the laser and the temperature of the MOF to obtain accurate windspeed measurements. ${ }^{7,10,11}$ In contrast, LGS must operate at frame rates of approximately

*mhart@optics.arizona.edu, +1-520-626-5265

Adaptive Optics Systems V, edited by Enrico Marchetti, Laird M. Close, Jean-Pierre Véran, Proc. of SPIE Vol. 9909, 99095N (C) 2016 SPIE $\cdot$ CCC code: $0277-786 \mathrm{X} / 16 / \$ 18 \cdot$ doi: $10.1117 / 12.2235080$ 
$1000 \mathrm{~Hz}$, and stability is required only at a level that maintains adequate return flux. To our knowledge, no measurements have been made of the achievable contrast from the resonant backscatter during the day with a view to establishing the viability of its use as a LGS. We report such measurements in this paper, and explore the implications for $\mathrm{AO}$ wave-front sensing on an ELT.

\section{DESCRIPTION OF THE EXPERIMENT}

The University of Arizona (UA) has a sodium laser on long-term loan from the US Air Force Research Laboratory. The laser generates approximately $6.5 \mathrm{~W}$ of narrow-band continuous-wave light in a beam that is circularly polarized to take advantage of optical pumping in the mesosphere to enhance the return flux. ${ }^{12}$ It is installed on an optical bench next to the UA's 1.5 m Kuiper telescope on Mount Bigelow in Arizona. The beam has a gaussian profile and is launched through a $10 \mathrm{~cm}$ aperture that is separated from the Kuiper telescope's optical axis by approximately $5 \mathrm{~m}$. For the experiment described in this paper, the laser launch projector was pointed at zenith, and the Kuiper telescope pointed slightly off zenith to place the sodium resonance beacon at about $90 \mathrm{~km}$ range in the center of its field of view. A simple instrument bolted to the Cassegrain focus of the Kuiper telescope included an imaging camera behind a sodium MOF from the Eddy Company (Apple Valley, California). We used this arrangement to evaluate the potential for using a sodium LGS for daytime wave-front sensing.

The basic MOF consists of a cell that contains a sodium atomic vapor placed between crossed linear polarizers in a kilogauss-level magnetic field. The magnetic field results in both Zeeman splitting of the atomic resonance lines in the vapor and a variation of the polarization-dependent absorption coefficient and refractive index (anomalous dispersion) with wavelength across the vapor absorption lines. A suitable choice of vapor temperature and magnetic field strength results in the absorption and birefringence profiles producing wavelength-dependent transmission in the vicinity of the resonance lines through rotation of the polarization. ${ }^{13}$ At the wavelength of peak transmission, a linearly polarized beam with axis aligned to the first polarizer will be rotated in the vapor cell to align precisely with the axis of the second polarizer. Under these conditions, the theoretical transmission maximum for the MOF configuration used in the work reported here (longitudinal magnetic field strength $3.7 \mathrm{kG}$, temperature $167{ }^{\circ} \mathrm{C}$ ) is close to $100 \%$ (Figure 1), without accounting for transmission losses in the optics. To maximize the LGS signal at our detector we placed a hightransmission $(\mathrm{T}=0.995)$ zero-order quarter-wave plate (QWP) in front of the MOF to convert the circularly polarized return laser light into linearly polarized light with the axis aligned to the first polarizer of the MOF.

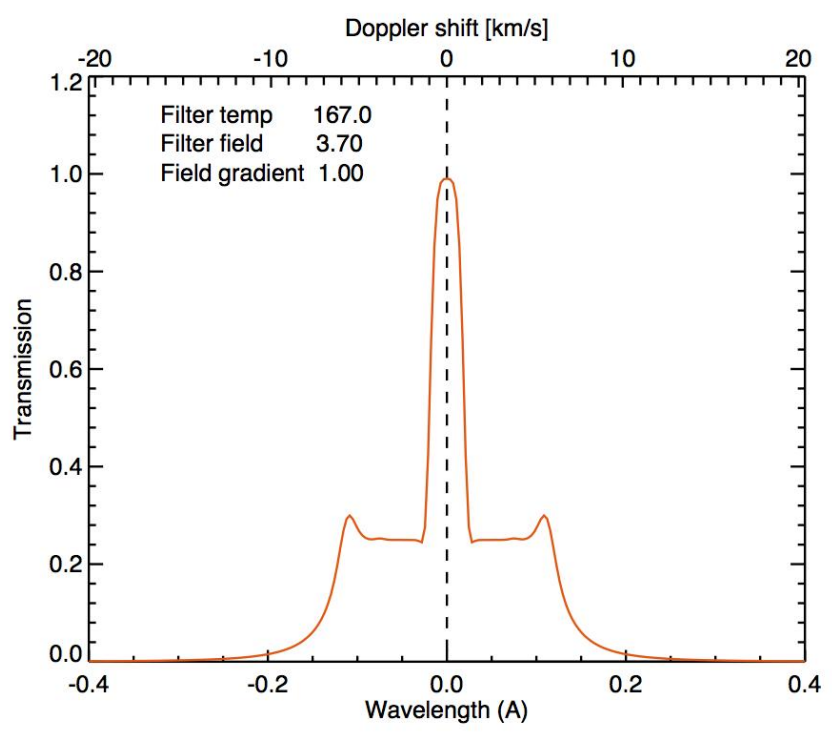

Figure 1. Theoretical transmission curve for linearly polarized light at the entrance window of an MOF with a longitudinal magnetic field strength of $3.7 \mathrm{kG}$, operating at a temperature of $167^{\circ} \mathrm{C}$.

Practically, a narrow-band interference filter is required in front of the MOF to suppress off-band leakage through the polarizers. Figure 2 shows the measured transmission of the optics of our MOF with the vapor cell cold and polarizers uncrossed: the width of the profile in this condition is determined by the interference filter. The peak at 0.5 is 
commensurate with the manufacturers' transmission values for the components: 0.82 for the polarizers, 0.75 for the narrow-band interference filter, and 0.995 for each of the windows of the sodium vapor cell. We note that polarizers and interference filters with higher transmission values are available which would increase the transmission of the MOF optics to 0.76 . For detection we use a Point Grey Flea3 camera in 12 bit mode. To allow us to compute absolute photometry of the LGS, we determined the mean gain of the camera by measuring a photon transfer curve. ${ }^{14}$ The result of $2.96 \mathrm{e}-/ \mathrm{ADU}$ is in good agreement with the manufacturer's specification of $3.0 \mathrm{e}-/ \mathrm{ADU}$.

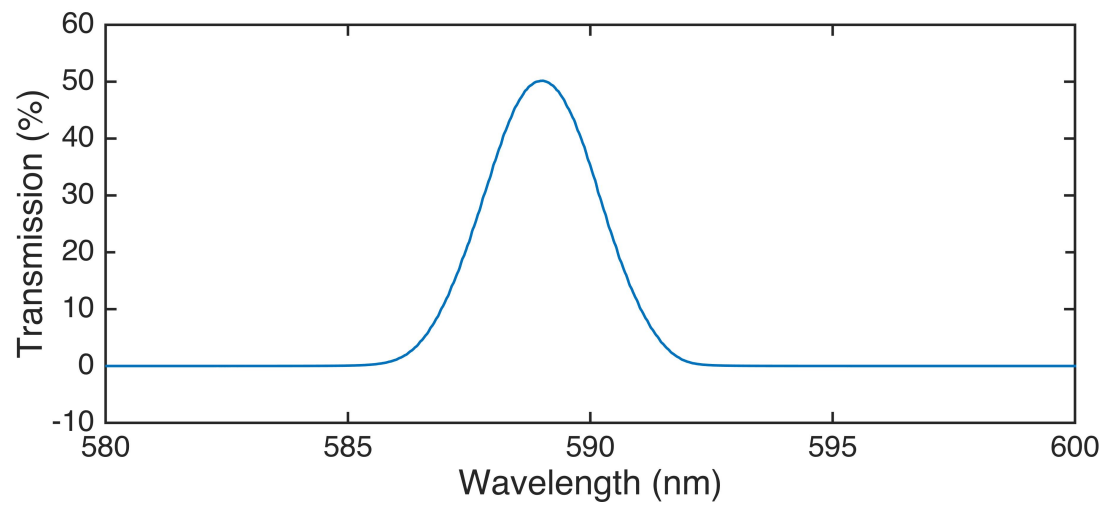

Figure 2. Transmission curve of the cold MOF with uncrossed polarizers. The profile is determined by the interference filter ahead of the MOF itself.

After acquiring the LGS on the imaging camera, we focused the $1.5 \mathrm{~m}$ telescope at the height of the mesospheric sodium layer. Continuous sequences of 200-500 images were recorded at 4 frames per second with individual exposure times of $0.25 \mathrm{~s}$. Figure 3 shows the LGS seen through the MOF in an integration of $8 \mathrm{~s}$, the sum of 32 exposures, recorded during daylight, approximately 42 minutes after sunrise. The LGS has a full width at half maximum (FWHM) of 3.1 arcsec, which is the result of a combination of seeing and diffraction of the gaussian beam profile at the launch aperture. The image scale on our camera was $0.156 \mathrm{arcsec} / \mathrm{pixel}$, which for analysis was binned by $16 \times$ to $2.5 \mathrm{arcsec} / \mathrm{pixel}$.

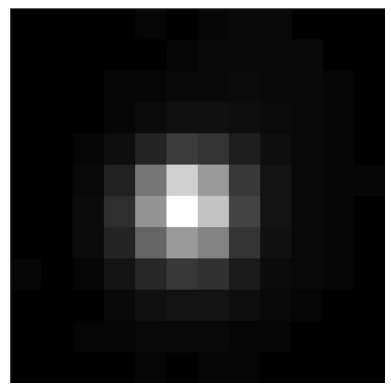

Figure 3. The LGS seen against the daytime sky at 07:04 local time. The exposure time is $8 \mathrm{~s}$ and the pixel scale is 2.5 arcsec.

We observed the characteristics of the LGS from 00:00 local time (07:00 UTC) to 07:04 local time (14:04 UTC) on March 24, 2016. As local sunrise was at 06:22 (13:22 UTC) this provided observations during both nighttime and daytime conditions. The change in contrast between the laser light and the background sky is nicely illustrated in the photographs in Figure 4.

\section{RESULTS}

The MOF transmission was estimated from nighttime sequences of LGS images with and without the MOF installed in the optical beam from the telescope. Maximum transmission was ensured by careful alignment of the QWP and polarizer axes and by adjusting the MOF operating temperature. The measured fluxes with and without the MOF were respectively $8.4 \times 10^{5}$ and $1.90 \times 10^{6}$ photon $/ \mathrm{m} / \mathrm{s}$, implying a transmission for the whole MOF of 0.44 . This value is consistent with expectations when two additional sources of reduced transmission are included. First, a scan of the 

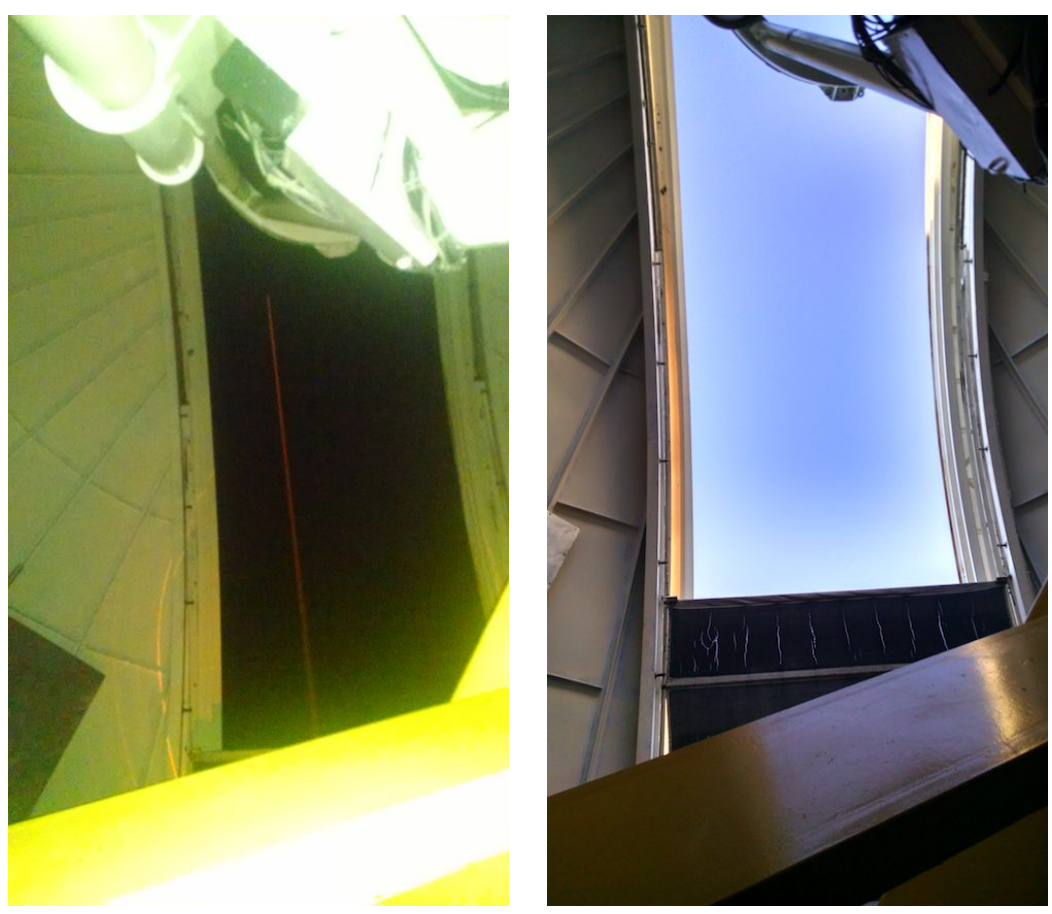

Figure 4. (Left) The laser beam propagating to zenith through the slit of the Kuiper telescope dome is easily seen at night. (Right) Approximately the same view in daylight when the beam is very hard to see.

wavelength of the laser revealed that the central passband of our MOF was shifted $0.001 \mathrm{~nm}$ from the wavelength of the sodium atoms in the mesosphere by the buffer gas used in the vapor cell. This reduced the transmission by an additional factor of 0.92. Second, the temperature of our narrowband interference filter was not stabilized and the difference between the ambient temperature in the telescope dome and the design operating temperature for the filter resulted in a $0.35 \mathrm{~nm}$ shift in the center passband, leading to a transmission reduction of 0.97 .

Table 1 shows the LGS and sky brightness as seen through the MOF as the sun rose. The LGS brightness was found to remain approximately constant over the time frame of these observations at a mean value of $1.20 \times 10^{6} \mathrm{photon} / \mathrm{m} / \mathrm{s}$. This is consistent with previous measurements ${ }^{15}$ given our output power at the time of approximately $6.5 \mathrm{~W}$, the low $\mathrm{Na}$ column density of $2.5 \times 10^{9} \mathrm{~cm}^{-2}$ expected in early spring, about half the annual mean, ${ }^{16}$ and the overall transmission of the system which we estimate to be 0.19 . The telescope itself, with two bare aluminum-coated mirrors, has a throughput of $\sim 0.8$; the relay optics, comprising two protected silver fold mirrors, an AR-coated collimating lens and the QWP, contribute a factor of 0.95 , the measured transmission of the MOF is 0.44 , and the Flea3 quantum efficiency at $589 \mathrm{~nm}$ is quoted by the manufacturer to be 0.56 . This would imply a flux at the telescope aperture of $1.0 \times 10^{6} \mathrm{photon} / \mathrm{m} / \mathrm{s} / \mathrm{W}$, although we note that the uncertainty in this estimate is unquantified.

The results of Table 1 are shown graphically in Figure 5. The sky background remains very low throughout the period of the observations at about $1000-1100$ photon $/ \mathrm{m}^{2} / \mathrm{s} / \operatorname{arcsec}^{2}$ with a slight rise toward the end of the sequence, about 45 minutes after sunrise. Unfortunately, observations had to cease at that time to avoid subjecting the telescope head ring to direct sunlight.

\section{WAVE-FRONT SENSING FOR ELTS}

From these quantitative measurements of LGS flux and sky background through the MOF we can calculate the expected signal-to-noise ratio (SNR) for a WFS operating during the day on an ELT. We expect some loss of signal because of the imperfect transmission of the filter, and the photon noise from background illumination will certainly be higher than at night. We note, however, that most astronomical AO systems are designed to correct wave fronts in bands down to the near IR, at $2 \mu \mathrm{m}$ or shorter, including the laser-guided systems for the three ELTs, while daylight observing at those telescopes is likely to be restricted to bands at $3.5 \mu \mathrm{m}$ and longer. In this regime, the AO system requirements on speed 
Table 1. LGS and Sky Brightness Results

\begin{tabular}{|c|c|c|}
\hline $\begin{array}{l}\text { Sequence } \\
\text { start time }\end{array}$ & $\begin{array}{c}\text { Sky brightness } \\
\text { (photon } \mathrm{m}^{-2} \mathrm{~s}^{-1} \operatorname{arcsec}^{-2} \text { ) }\end{array}$ & $\begin{array}{l}\text { LGS brightness } \\
\left(\text { photon } \mathrm{m}^{-2} \mathrm{~s}^{-1}\right)\end{array}$ \\
\hline $06: 33: 33$ & 961 & $1.27 \times 10^{6}$ \\
\hline $06: 50: 38$ & 957 & $1.18 \times 10^{6}$ \\
\hline $06: 52: 49$ & 1050 & $1.14 \times 10^{6}$ \\
\hline $06: 54: 58$ & 1060 & $1.22 \times 10^{6}$ \\
\hline $06: 57: 07$ & 1090 & $1.26 \times 10^{6}$ \\
\hline $06: 59: 17$ & 1070 & $1.12 \times 10^{6}$ \\
\hline $07: 01: 36$ & 1040 & $1.19 \times 10^{6}$ \\
\hline 07:03:50 & 1080 & $1.18 \times 10^{6}$ \\
\hline
\end{tabular}
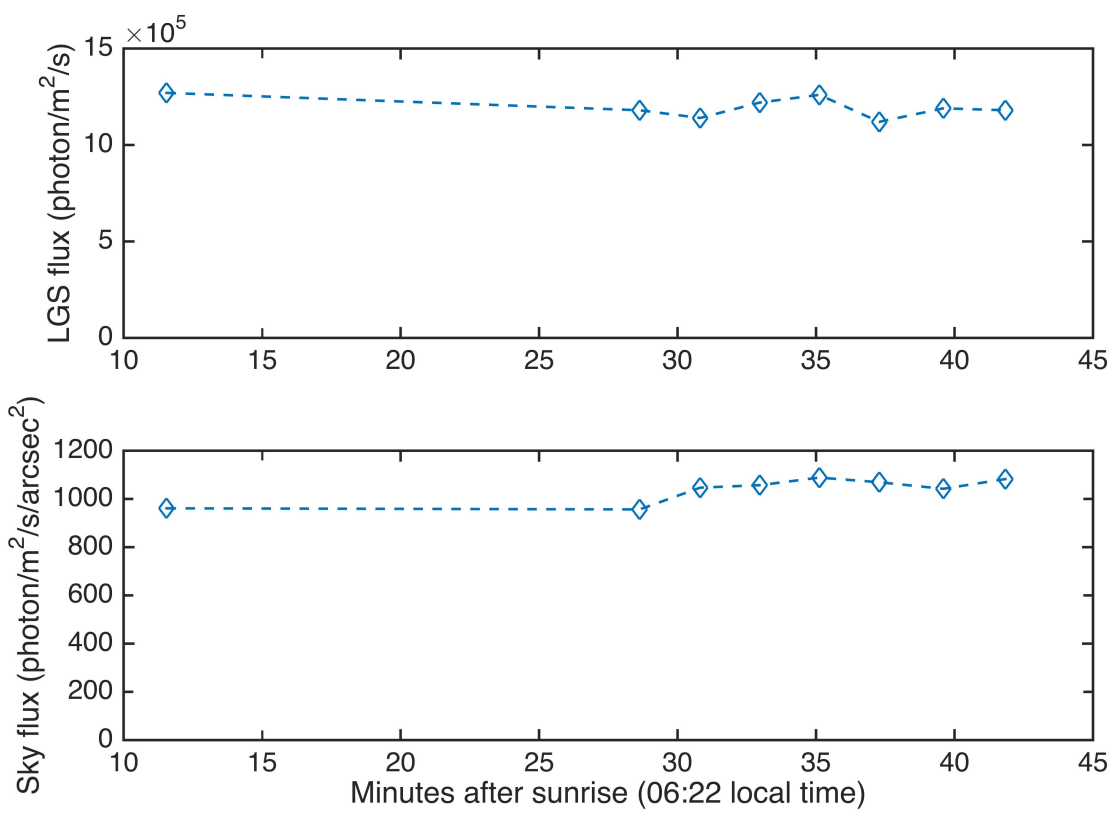

Figure 5. (Top) Flux from the LGS in the period after sunrise. (Bottom) Simultaneous measurements of the background flux from the sky.

and spatial sampling of the wave front are reduced, so lost SNR may, if necessary, be recovered by longer integrations or larger subapertures on the WFS.

As a case study, we consider the LGS WFS of the TMT's Narrow Field InfraRed AO System (NFIRAOS). ${ }^{4}$ While the imaging sensors in NFIRAOS are only projected to extend out to the $\mathrm{K}$ band, with no provision for the thermal IR, the design is mature and representative of the capabilities of laser-guided AO systems to be expected on the ELTs. NFIRAOS will deploy six Shack-Hartmann type WFS, with square subapertures $0.5 \mathrm{~m}$ on a side, running nominally at 800 frames per second. Each WFS sees the return from a single sodium LGS generated by a laser of approximately 20 W. Although the lasers are launched from behind the telescope's secondary mirror, parallax causes the image of the LGS to be elongated by as much as 4 arcsec as seen by subapertures at the outer edge of the pupil. The field of view of these subapertures is therefore rather large at about $3 \times 7.5$ arcsec. Assuming, rather pessimistically, an overall photon efficiency not significantly different than we saw at the Kuiper telescope, the laser flux during the day would be 1150 
photon per subaperture per frame, and the mean sky flux through the MOF would be just 7.7 photon per subaperture per frame. This is comparable to the unfiltered flux that would be seen from a sky of visual magnitude $18 \operatorname{arcsec}^{-2}$, conditions typical of a dark site at night under a full moon. The uncertainty in the WFS signal would therefore be dominated by the shot noise from the LGS, the desirable regime in which to operate. Assuming read noise of 3 e- rms from the WFS pixels, the SNR per subaperture would be about 25.

At $3.5 \mu \mathrm{m}$, the shortest wavelength where daytime operation at the ELTs might be considered, characteristic nighttime values of the Fried parameter $r_{0}$ will be about $1.5 \mathrm{~m}$. During the day, however, the seeing may worsen by a factor of 2-3, leading to $r_{0} \sim 0.5 \mathrm{~m}$, or $D / r_{0} \sim 60$, where $D$ is the telescope diameter. Achieving good AO correction requires that the SNR on the WFS be approximately equal to $D / r_{0}$ so that wave-front slopes may be estimated with an uncertainty no greater than the angular size of a speckle in the full-aperture focal plane. Ideally, then, we would prefer SNR of 60 rather than 25. To achieve this would require a flux from the LGS of 4350 photon per subaperture per frame, nearly four times higher than our calculation predicts. We believe this can be achieved by a combination of improved throughput and slightly slower operating speeds for the AO system. As we noted above, the transmission of the MOF can be improved by a factor of 1.7 by selecting better (and more expensive) components and by more careful thermal regulation. The TMT's WFS detectors have a quantum efficiency that is $50 \%$ higher at $589 \mathrm{~nm}$ than the camera used in our experiments. Even without higher transmission for the telescope and instrument optics than we achieved, which may be challenging given the complexity of TMT and NFIRAOS, the required flux could then be reached by running the AO system at 500 $\mathrm{Hz}$ rather than $800 \mathrm{~Hz}$. This is not an unreasonable compromise at the long wavelengths we are considering: although the seeing is substantially worse during the day than at night, the coherence time, set by fast winds at high altitude, does not degrade as much since the strongest diurnal effect is solar heating of the ground near the telescope. Similar considerations apply to AO instruments on the other ELTs as well, such as ELT-MIDIR, the mid-IR imager planned as a second-light instrument on the E-ELT. ${ }^{17}$

Of course, as with any laser-guided AO system, at least one natural star is required to sense tip-tilt. We believe this will be best done in the thermal IR bands so that the star images will benefit from sharpening to the diffraction limit by the AO. This is an enormous advantage for an ELT in achieving satisfactory visibility of the star against the thermal emission from the daytime sky. At a good astronomical site, the daytime sky surface brightness at $3.5 \mu \mathrm{m}$ is about magnitude 5 per sq. arcsec or approximately $1.5 \times 10^{7}$ photon $/ \mathrm{m}^{2} / \mathrm{s} / \mu \mathrm{m} / \operatorname{arcsec}^{2}{ }^{1}$ Assume the AO system is able to correct to $80 \%$ Strehl ratio in $\mathrm{M}$ band in short exposures, given the poor daytime seeing, implying a tilt-removed wave-front error of $260 \mathrm{~nm} \mathrm{rms}$, and that we wish a Strehl ratio degradation of no more than $10 \%$ from tip-tilt errors. That requires an all-source tilt residual of $<180 \mathrm{~nm} \mathrm{rms}$, corresponding to $0.2 \lambda / D$. Tilt measurements on the diffraction-limited star image must be made therefore at a SNR of at least 5 , leading to a star brightness requirement of $1400 \mathrm{photon} / \mathrm{m}^{2} / \mathrm{s} / \mu \mathrm{m}$, or an apparent M-band magnitude of about 14.5. ${ }^{19}$ Averaged over Galactic coordinates, there are about 1000 such stars per square degree in the sky. ${ }^{20}$ The isokinetic angle, over which tip-tilt is approximately constant, scales with telescope diameter and for a $30 \mathrm{~m}$ telescope is about $300 \operatorname{arcsec}$ at $3.5 \mu \mathrm{m} .{ }^{21}$ We would therefore expect about 7 stars of the required brightness per isokinetic patch, sufficient to ensure essentially complete sky coverage. Any region of the sky that does not have telescope pointed near the sun will be accessible to AO imaging in the thermal IR bands.

\section{REFERENCES}

[1] J. M. Beckers and A. Cacciani, "Using laser beacons for daytime adaptive optics," Exp. Astr. 11, 133-143 (2001).

[2] E. Diolaiti et al., "Conceptual design and performance of the multiconjugate adaptive optics module for the European Extremely Large Telescope," Proc. SPIE 7736, 77360R (2010).

[3] A. H. Bouchez et al., "The Giant Magellan Telescope adaptive optics program," Proc. SPIE 9148, 91480W (2014).

[4] C. Boyer et al., "Adaptive optics program at TMT," Proc. SPIE 9148, 91480X (2014).

[5] A. Cacciani and A. M. Fofi, "The magneto-optical filter II: Velocity field measurements," Sol. Phys. 59, 179-189 (1978).

[6] H. Chen et al., "Daytime operation of Na temperature LIDAR using a dispersive faraday vapor filter," Proc. SPIE 2833, 46- 52 (1996). 
[7] C. Y. She et al., "The first 80-hour continuous LIDAR campaign for simultaneous observation of mesopause region temperature and wind," Geophys. Res. Lett. 30, doi:10.1029/2002GL016412 (2003).

[8] T. Pfrommer, P. Hickson, and C. Y. She, "A large-aperture sodium fluorescence lidar with very high resolution for mesopause dynamics and adaptive optics studies," Geophys. Res. Lett. 36, doi:10.1029/2009GL038802 (2009).

[9] X. Hu et al., "Sodium fluorescence doppler LIDAR to measure atmosphericr temperature in the mesopause region," Chin. Sci. Bull. 56, 417-423 (2011).

[10] C. Y. She et al., "High-spectral-resolution fluorescence light detection and ranging for mesospheric sodium temperature measurements," Appl. Opt. 31, 2095-2106 (1992).

[11] Y. Yang et al., “A flat spectral faraday filter for sodium lidar,” Opt. Lett. 36, 1302-1304 (2011).

[12] J. C. Bienfang et al., " $20 \mathrm{~W}$ of continuous-wave sodium D2 resonance radiation from sum-frequency generation with injection-locked lasers," Opt. Lett. 28, 2219-2221 (2003).

[13] W. Kiefer et al., "Na-Faraday rotation filtering: The optimal point," Nature Scientific Reports 4, 1-7 (2014).

[14] J. R. Janesick, K. P. Klaasen, and T. Elliott, "Charge-coupled-device charge-collection efficiency and the photontransfer technique," Opt. Engr. 26, 972-980 (1987).

[15] J. Ge et al.,"Simultaneous measurements of sodium column density and laser guide star brightness," Proc. SPIE 3353, 242-253 (1998).

[16] G. C. Papen, C. S. Gardner, and G. Yu, "Characterization of the mesospheric sodium layer," OSA Technical Digest Series 13, 96-99 (OSA, Washington, DC) (1996).

[17] B. Brandl et al., "MIDIR/T-OWL: the thermal/mid-IR instrument for the E-ELT," Proc. SPIE 8446, 626920 (2006).

[18] P. J. McGregor et al., "GMT Integral-Field Spectrograph (GMTIFS) conceptual design," Proc. SPIE 8446, 84461I (2012).

[19] Gemini Observatory flux converter at https://www.gemini.edu/?q=node/11119

[20] J. N. Bahcall and R. M. Soneira, "The Universe at faint magnitudes. I. Models for the Galaxy and the predicted star counts," Astrophys. J. Suppl. 44, 73 (1981).

[21] J. W. Hardy, “Adaptive optics for astronomical telescopes,” (Oxford: OUP) (1998). 